\title{
Nondestructive Evaluation of Flaws Using SQUIDs
}

\author{
J. T. Jeng ${ }^{a}$, H. E. Horng ${ }^{a}$, Chao-Hsiang Chen ${ }^{b}$, J. H. Chen ${ }^{b}$, and H. C. Yang ${ }^{b}$ \\ ${ }^{a}$ Department of Physics, National Taiwan Normal University, Taipei 116, Taiwan \\ ${ }^{b}$ Department of Physics, National Taiwan University, Taipei 106, Taiwan
}

Using high- $T_{c}$ SQUIDs, we have designed a nondestructive evaluation system to probe buried flaws. The frequency of the excitation magnetic fields was varied from 10 to $800 \mathrm{~Hz}$ and the magnetic fields generated from the eddy current was lock-in detected. The SQUIDs output data revealed the most effective excitation frequencies for the flaws with different depths and widths. The signal-to-noise ratio of the deep flaws can be further enhanced either by using higher excitation fields or using the SQUIDs with higher low-frequency sensitivity.

\section{INTRODUCTION}

The low-noise high- $T_{c}$ SQUIDs are valuable for application in nondestructive evaluation (NDE) for its unparalleled sensitivity [1-3]. For a conductive sample, the eddy current SQUID NDE technique is more suitable than the ultrasonic technique when a good acoustical coupling with the sample is not available. In order to probe the deeper flaws with the eddy current NDE system, one may use the most effective excitation frequency [2] to obtain higher signal-to-noise ratio. In this work, we present the results of the most effective excitation frequencies to enhance the signal-to-noise ratio of the NDE system.

\section{EXPERIMENTAL DETAILS}

The sensor of the NDE system [4] was a dc SQUID magnetometer controlled by the SQUID electronics (Conductus Inc. Model IMC 303). The magnetometer was mounted inside a liquid-nitrogen fiberglass dewar shielded with a $\mu$-metal cylinder to reduce environmental noise. A double-D excitation coil was mounted beneath the dewar and the diameter of the coil was $28 \mathrm{~mm}$. The position of the double-D coil was adjusted to minimize the excitation field in the SQUID. The distance between the excitation coil and the SQUID was about $10 \mathrm{~mm}$ while the distance between the excitation coil and the sample was $3 \mathrm{~mm}$. The sample was mounted on a computer-controlled $x-y$ table. The alternating magnetic field was applied by a function generator and the excited signal sensed by the SQUID was detected by a lock-in detector (Stanford Research, Model SR530).

\section{RESULTS AND DISCUSSION}

The peak-to-peak magnetic field $\Delta \mathrm{B}_{\text {slot }}$ detected by the SQUID from a $4.2 \mathrm{~mm}$-deep slot at different excitation frequencies is shown in Figure 1. The corresponding field signal from the edge of the stack

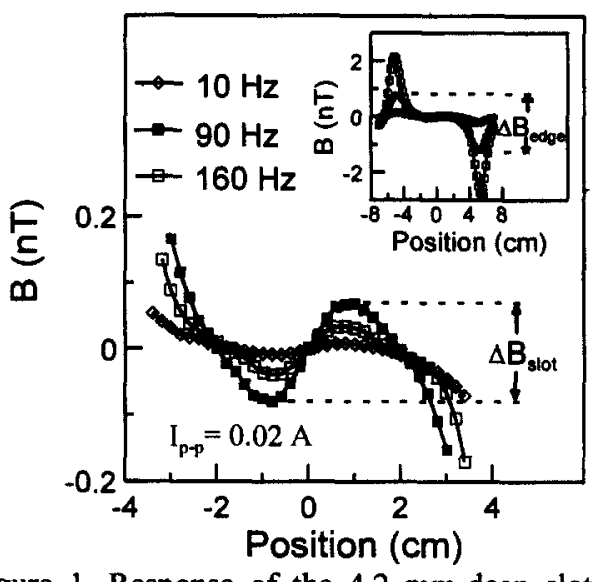

Figure 1. Response of the $4.2 \mathrm{~mm}$-deep slot at excitation frequencies of 10,90 and 160 Hz. $\Delta \mathrm{B}_{\text {slot }}$ and $\Delta \mathrm{B}_{\text {edge }}$ for the excitation frequency of $90 \mathrm{~Hz}$ are indicated in the figure and in the inset respectively. 


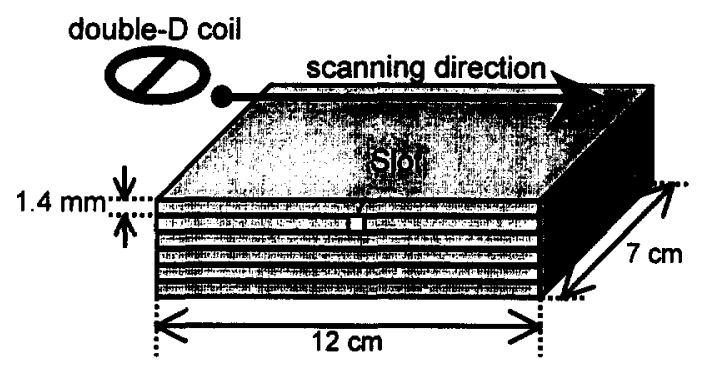

Figure 2. A slot covered with aluminum plates to simulate a buried crack. The scanning direction and the alignment of the double-D coil are also shown here.

$\Delta B_{\text {edge }}$ is shown in the inset of Figure 1. The sample was a stack of aluminum plates with a slot of width $2 \mathrm{~mm}$ in one plate to simulate a buried crack. The geometry of the sample is shown in Figure 2. Both $\Delta B_{\text {slot }}$ and $\Delta B_{\text {edge }}$ were found to be weak at the excitation frequency of $10 \mathrm{~Hz}$. At the higher frequencies of 90 and $160 \mathrm{~Hz}, \Delta \mathrm{B}_{\text {slot }}$ increases at first and then decreases.

The frequency dependences of $\Delta \mathrm{B}_{\text {slot }}$ and $\Delta \mathrm{B}_{\text {edge }}$ for the slot at different buried depths are shown in Figure 3. The frequency dependence of $\Delta \mathrm{B}_{\text {edge }}$ at the edge is shown in the inset. It was found that $\Delta \mathrm{B}_{\text {edge }}$ increased monotonically with increasing frequency up to $800 \mathrm{~Hz}$. On the other hand, the most effective excitation frequencies $f_{\mathrm{m}}$ [2] for $\Delta \mathrm{B}_{\text {slot }}$ were found to be at 200,120 , and $70 \mathrm{~Hz}$ respectively for buried slots of depth $1.4,2.8$ and $4.2 \mathrm{~mm}$. In other words, the value of $f_{\mathrm{m}}$ for the slot is lower as the slot is deeper. Besides, the value of $f_{\mathrm{m}}$ for a buried slot of $0.6-\mathrm{mm}$ wide was found to be $120 \mathrm{~Hz}$ in contrast to $200 \mathrm{~Hz}$ for the $2 \mathrm{~mm}$-wide slot at the same buried depth of $1.4 \mathrm{~mm}$ as shown in the dashed line of Figure 3. Hence, for the same slot depth, $f_{m}$ is lower if the width of the buried slot is narrower. We note that the value of $\Delta \mathrm{B}_{\text {slot }}$ is $0.2 \mathrm{nT}$ for a buried slot of depth $4.2 \mathrm{~mm}$ and width $2 \mathrm{~mm}$ excited at an optimal frequency of $70 \mathrm{~Hz}$. The noise level of the SQUID in an unshielded environment is $0.02 \mathrm{nT} / \mathrm{Hz}^{\prime 2}$ at 70 $\mathrm{Hz}$. Although one may use a lower $f_{\mathrm{m}}$ to optimize $\Delta B_{\text {slot }}$ for the deeper slots, the $\Delta B_{\text {slot }}$ becomes weaker for the deeper slots. In this case, the noise level of the SQUIDs at the low frequencies may exceed the detected signal $\Delta \mathrm{B}_{\text {slot. }}$ Therefore, a SQUID with

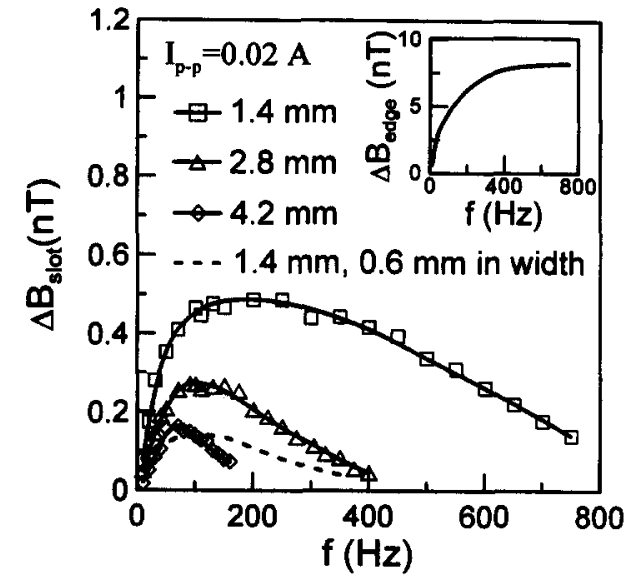

Figure 3. $\Delta \mathrm{B}_{\text {slot }}$ at various excitation frequencies for the $2 \mathrm{~mm}$ slot at the depth of 1.4 , 2.8 , and $4.2 \mathrm{~mm}$. The dash curve is the result for a $0.6 \mathrm{~mm}$-wide, $1.4 \mathrm{~mm}$-deep slot. The inset is $\Delta \mathrm{B}_{\text {edge }}$ of the stack at various excitation frequencies

better low frequency noise level is essential in order to probe deeper slots.

\section{CONCLUSION}

In summary, the most effective excitation frequency $f_{\mathrm{m}}$ for the SQUID NDE system depends on the depth and the width of the crack. For a deeper crack, the $f_{\mathrm{m}}$ is found to be lower. Besides, the higher the noise resolution at low frequencies, the better the signal to noise ratio the SQUID NDE system can have.

\section{REFERENCES}

1. H.Weinstock, IEEE Trans. Mag. 27 (1991) 3231.

2.M.V.Kreutzbruck, J.Troll, M.Muck, C.Heiden, and Y.Zhang, IEEE Trans. Appl. Supercond., 7 (1997) 3279.

3.C.Carr, D.McA.Mckirdy, E.J.Romans, G.B. Donaldson and A.Cochran, IEEE Trans. Appl. Supercond., 7 (1997) 3275.

4.J.T.Jeng, H.E.Horng, Chao-Hsiang Chen, J.H. Chen, and H.C.Yang, presented at the "4th European Conference on Applied Superconductivity", Sep. 14-17, 1999. 